\title{
POSTURAS DE TRABAJO Y EL NIVEL DE RIESGO PARA DESARROLLAR UNA ENFERMEDAD OCUPACIONAL EN LOS ESTUDIANTES DE ODONTOLOGÍA DE LA UNIVERSIDAD CATÓLICA DE CUENCA -ECUADOR 2016
}

\section{WORK POSTURE AND THE RISK LEVEL TO DEVELOP AN OCCUPATIONAL DISEASE IN DENTISTRY STUDENTS OF THE CATHOLIC UNIVERSITY OF CUENCA -ECUADOR 2016}

\author{
Correa-Carrera Katherine ${ }^{1 *}$, Sánchez-Zamora Rene ${ }^{2}$, Villavicencio-Caparó Ebingen ${ }^{3}$, Granda-Songor Mayra ${ }^{1}$ \\ ${ }^{1}$ Odontóloga General, práctica privada.Ecuador \\ 2 Doctor en Medicina y Cirugía- Magíster en Seguridad, Salud y Ambiente Universidad Católica de Cuenca.Ecuador. \\ ${ }^{3}$ Doctor en Ciencias de la Salud - Bioestadístico, Universidad Católica de Cuenca. Ecuador. \\ *kthy7c@gmail.com
}

\begin{abstract}
Resumen
OBJETIVO: Evaluar el nivel de riesgo de una posición inadecuada de trabajo de los estudiantes de Quinto año de la Carrera de Odontología de la Universidad Católica de Cuenca- Ecuador 2016. MATERIALES Y MÉTODOS: Estudio transversal de evaluación visual,la población fue de 185 estudiantes,se incluyeron 125 estudiantes que realizaban continuamente sus prácticas pre profesionales, fueron excluidos los estudiantes que no aportaron con los datos personales en las fichas de investigación. Se utilizó los criterios de evaluación proporcionados por el Método REBA evaluando el nivel de riesgo de la postura de trabajo, el estudiante firmó un consentimiento informado, el examen visual fue realizado en la Clínica Integral "Cesar Cordero Moscoso". RESULTADOS: La muestra presentó un 35,2\% (44) correspondiente al sexo masculino y un 64,8\% (81) correspondientes al sexo femenino. En los 125 estudiantes se determinó que el $61.6 \%$ (77) posee un nivel de riesgo medio, por lo tanto su intervención es necesaria; así mismo se encontró que el $37.6 \%$ (47) presentó un nivel de riesgo bajo y su intervención puede ser necesaria; en menor porcentaje se encontró que el $0.8 \%$ (1) tiene un nivel de riesgo inapreciable por lo tanto es la única persona en la que la intervención no es necesaria; en cuanto al nivel de riesgo alto o muy alto no se encontró ningun estudiante con estas características.
\end{abstract}

Palabras clave: Riesgo laboral, dolor postural, músculo esquelético, odontología.

\begin{abstract}
AIM: The sample was calculated using a formula based on a population of 185 students. The students that were continuously carrying out carried out their preprofessional interships were included, students who did not contribute with the personal data were excluded.MATERIALS AND METHODS: The evaluation criteria provided by the REBA Method was used evaluating the level of risk of the work posture, the student signed an informed consent, the visual examination was performed at the "Cesar Cordero Moscoso" Comprehensive Clinic.RESULTS: The sample had a 35.2\% (44) corresponding to the male sex and a $64.8 \%$ (81) corresponding to the female sex. In the 125 students it was determined that 61.6\% (77) had an average level of risk, therefore their intervention is necessary; it was also found that $37.6 \%$ (47) presented a low level of risk and their intervention may be necessary; in a lower percentage it was found that $0.8 \%$ (1) has an inestimable risk level, therefore it is the only person in whom the intervention is not necessary; in terms of high or very high risk level, no student with these characteristics was found.
\end{abstract}

Key words: Occupational Risk, posture pain, skeletal muscle, dentistry.

\section{INTRODUCCIÓN}

Una postura de trabajo incorrecta y conservada de forma prolongada, en conjunto con los años de práctica originará un 
riesgo para el profesional, en consecuencia se desarrollarán patologías en el sistema músculo esquelético. ${ }^{1}$ En la actualidad, las posturas inadecuadas y movimientos incorrectos se realizan para conseguir una mejor visibilidad del campo operatorio, llevando al profesional de la salud al cansancio físico y mental. ${ }^{1}$ El Odontólogo debe trabajar en una posición adecuada, los movimientos de poca amplitud evitarán la fatiga y ayudarán a satisfacer las exigencias del ámbito laboral. ${ }^{2}$ Una postura correcta de trabajo se considera como el equilibrio entre los músculos, huesos y articulaciones, que protegen a las demás estructuras del cuerpo de posibles traumas, la postura correcta no es una situación completamente estática sino más bien dinámica, en búsqueda de una postura correcta es necesaria la presencia de un tono muscular apropiado en conjunto, con la flexibilidad. ${ }^{3}$ La postura recomendada se denomina Posición de Máximo Equilibrio o Posición Cero, a nivel internacional se conoce como B.H.O.P (Balanced Human Operating Position), su objetivo es evitar tensión muscular y esqueletal, se caracteriza porque el odontólogo realiza su trabajo con la mayor cantidad de músculos en semi relajación. ${ }^{4}$ Una postura inadecuada es la causante de potenciales desórdenes músculo esqueléticos como por ejemplo:

- Flexión o extensión forzada de manos y brazos.

- Movimientos repetitivos en manos y brazos.

- Movimientos que implican de posturas extremas.

- Posturas estáticas prolongadas.

- Vibraciones.

- Permanecer de pie o sentados por periodos prolongados de tiempo.

- Posición de los brazos sobre la altura de los hombros.

- Cabeza en flexión o en rotación.

Este tipo de posiciones producen una acumulación progresiva de micro traumatismos, son de aparición lenta y en etapas tempranas son inofensivos, por lo que se ignora la presencia de síntomas hasta un estadio crónico y cuando el daño es permanente. ${ }^{4}$ En el cuello los movimientos de flexión, extensión, flexión lateral y rotación son más libres y amplios debido a los discos intervertebrales, a las cápsulas articulares que en esta zona son laxas, por lo tanto el movimiento de flexión es máxima en la región cervical, en cambio la extensión es más notoria que la flexión anterior en la región lumbar debido a que se incrementa la presión entre los espacios vertebrales. ${ }^{5}$ Dentro de la práctica profesional se ha podido encontrar que cinco de cada seis odontólogos experimentaron en alguna ocasión molestias y dolor en un periodo de 6 a 12 meses, siendo así el dolor de cuello y hombros las áreas de mayor molestia. ${ }^{6}$ Las diferentes posiciones prolongadas e inadecuadas nos llevan a dos tipos de dolor así tenemos:

- Dolor Agudo: produce una serie de efectos indeseables, como consecuencia de la llegada del estímulo doloroso a distintos niveles del sistema nervioso central, su duración de horas, días o semanas.

- Dolor Crónico: tiene una mayor duración puede ir desde los 3 a los 6 meses aún estando bajo tratamiento, la presencia de dolor agudo y crónico sin tratamiento puede llevar al desarrollo de alteraciones y patologías músculo esqueletales. ${ }^{7}$

Las enfermedades o trastornos músculo esqueléticos son un conjunto de lesiones inflamatorias de varias estructuras, su localización es más frecuente en cuello, espalda, hombros, codos, muñecas y manos, el síntoma con mayor predominio es el dolor que se asocia con inflamación, pérdida de fuerza, incapacidad o pérdida de la función. ${ }^{8}$ En la ciudad de Atlanta, el Instituto Nacional de Salud y Seguridad Ocupacional (NIOSH), indica que las lesiones o desordenes músculo esqueléticos, incluyen un grupo de condiciones que involucran a los nervios, tendones, músculos, y estructuras de apoyo como los discos intervertebrales, por lo tanto representan una amplia cantidad de desórdenes que pueden diferir en grado de severidad desde síntomas periódicos leves hasta condiciones crónicas severas. ${ }^{9}$ El diagnóstico del nivel de riesgo de una postura inadecuada la podemos realizar gracias al empleo del Método REBA (Rapid Entire Body Assessment), ${ }^{10}$ se caracteriza por ser un análisis visual que permite una valoración postural para riesgo músculo esquelético. Genera una puntuación para la actividad muscular debida a posturas estáticas, inestables y acciones repetitivas. Se caracteriza principalmente porque divide al cuerpo en dos grupos (Figura No. 1), el grupo A incluye tronco, cuello y piernas y el grupo $\mathrm{B}$ está formado por los brazos y las muñecas. ${ }^{10}$ Este método fue desarrollado por Hignett y McAtamney en NottinghamInglaterra en el año 2000, para estimar el riesgo de padecer desórdenes corporales relacionados con el trabajo. ${ }^{10}$ Para evitar el desarrollo de alguna enfermedad de tipo ocupacional se recomienda principalmente evaluar el puesto de trabajo, para evitar una postura de trabajo inadecuada , así también descansos cortos de 3 minutos entre cada paciente lo que mejorará el flujo sanguíneo, igualmente se recomienda descansos de recuperación que son de mayor duración, consisten en periodos de 10-15 minutos cada 2-3 horas de trabajo, estos descansos deben programarse a lo largo del día, que permitirán la recuperación completa de los músculos. ${ }^{11}$

\section{METODOLOGÍA}

Estudio transversal de evaluación visual, en una población de 185 estudiantes de Quinto Año de la Carrera de Odontología de la Universidad Católica de Cuenca-Ecuador, se incluyeron a 125 estudiantes $^{12}$ que realizaban continuamente sus prácticas pre profesionales, fueron excluidos los estudiantes que no aportaron con los datos personales en las fichas de investigación. Se utilizó los criterios de evaluación proporcionados por el Método REBA evaluando el nivel de riesgo de la postura de trabajo. El grupo A tiene un total de 60 combinaciones posturales para el tronco, cuello y piernas. La puntuación obtenida de la tabla A estará comprendida entre 1 y 9; a este valor se le debe añadir la puntuación resultante de la carga/fuerza cuyo rango esta entre 0 y $3 .{ }^{10} \mathrm{El}$ grupo B tiene un total de 36 combinaciones posturales para la parte superior 


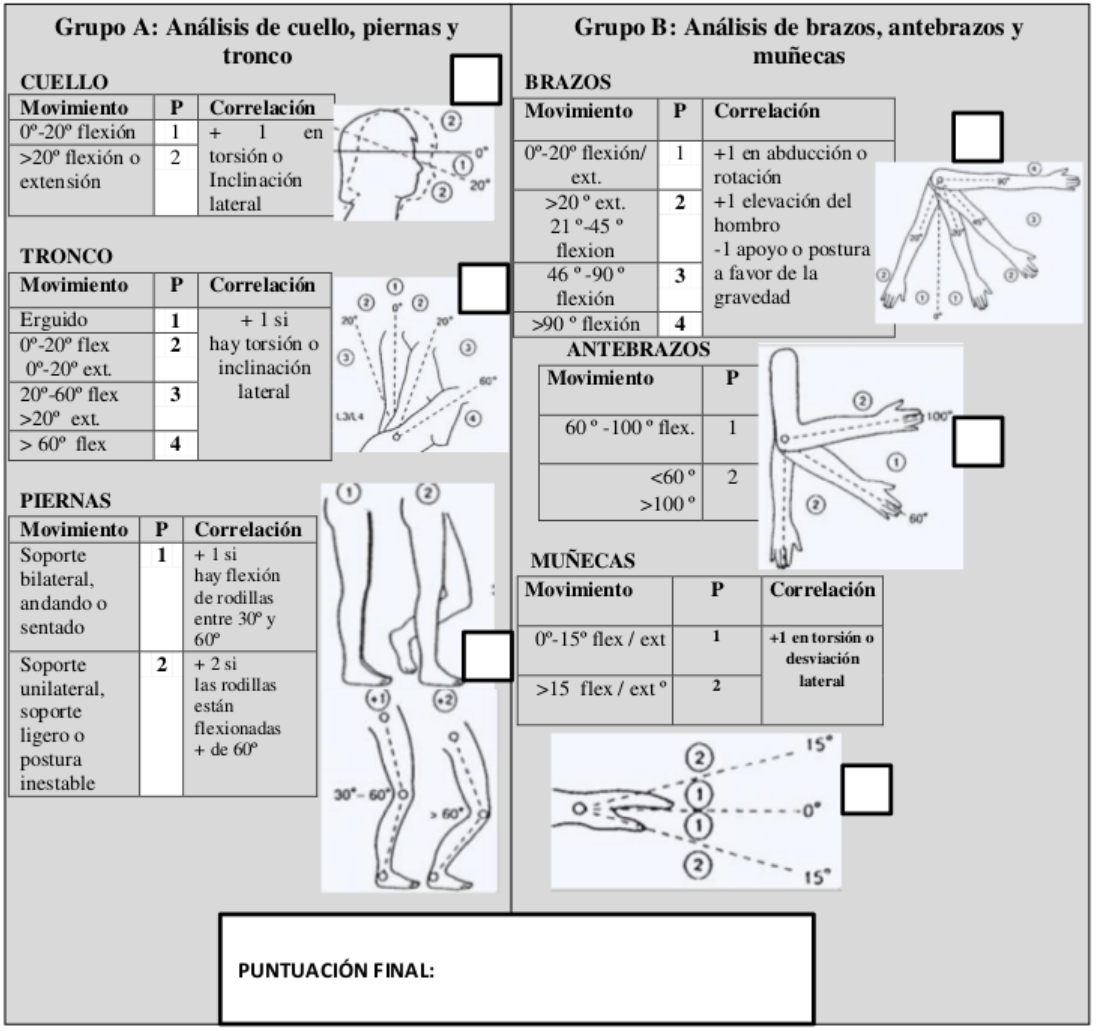

Fig. 1. Método REBA Grupo A - Grupo B

del brazo, parte inferior del brazo y muñecas, la puntuación final de este grupo, tal como se recoge en la tabla B, está entre 0 y 9; a este resultado se le debe añadir el obtenido de la tabla de agarre, es decir, de 0 a 3 puntos.

Los resultados A y B se combinan en la Tabla C (Figura No.2) para dar un total de 144 posibles combinaciones, y finalmente se añade el resultado de la actividad para dar el resultado final REBA que indicará el nivel de riesgo y el nivel de acción. ${ }^{10}$ La puntuación que hace referencia a la actividad (+1) se añade cuando:

- Una o más partes del cuerpo permanecen estáticas: por ejemplo, sostenidas durante más de 1 minuto.

- Repeticiones de una tarea: por ejemplo, más de cuatro veces por minuto (no se incluye el caminar).

- Acciones que causen grandes y rápidos cambios posturales.

- Cuando la postura sea inestable.

Puntuación final: A las 144 combinaciones posturales finales hay que sumarle las puntuaciones correspondientes al concepto de puntuaciones de carga, al acoplamiento y a las actividades; ello nos dará la puntuación final REBA que estará comprendida en un rango de 1-15, lo que nos indicará el riesgo que supone desarrollar el tipo de tarea analizada y nos indicará los niveles de acción necesarios en

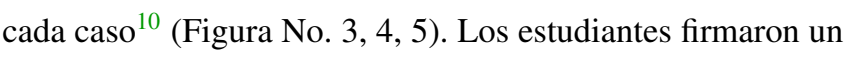
consentimiento informado, la toma de fotografías fueron de uso únicamente investigativo, la participación en el estudio fue de forma voluntaria; el examen visual fue realizado en la Clínica Integral "Cesar Cordero Moscoso" de la Carrera de Odontología de la Universidad Católica de Cuenca.

\section{RESULTADOS}

En los 125 estudiantes se aplicó el Método REBA (Gráfico No. 1) para determinar el nivel de riesgo de una postura, así se encontró que el $61.6 \%$ (77) posee un nivel de riesgo medio, por lo tanto su intervención es necesaria; así mismo se encontró que el $37.6 \%$ (47) presenta un nivel de riesgo bajo y su intervención puede ser necesaria; en menor porcentaje encontramos que el $0.8 \%$ (1) tiene un nivel de riesgo inapreciable, por lo tanto es la única persona en que la intervención no es necesaria; en cuanto al nivel de riesgo alto o muy alto no se encontró ningún estudiante con estas características.

También se valoró el nivel de riesgo de una postura según el sexo (Gráfico No. 2), se encontró en el sexo femenino que solo el $1 \%$ (1) de mujeres tuvo un nivel de riesgo inapreciable, seguido de un $33 \%$ (27) de mujeres que tuvo un nivel de riesgo bajo y un $65 \%$ (53) de mujeres que 
TABLAS DE CALIFICACIÓN:

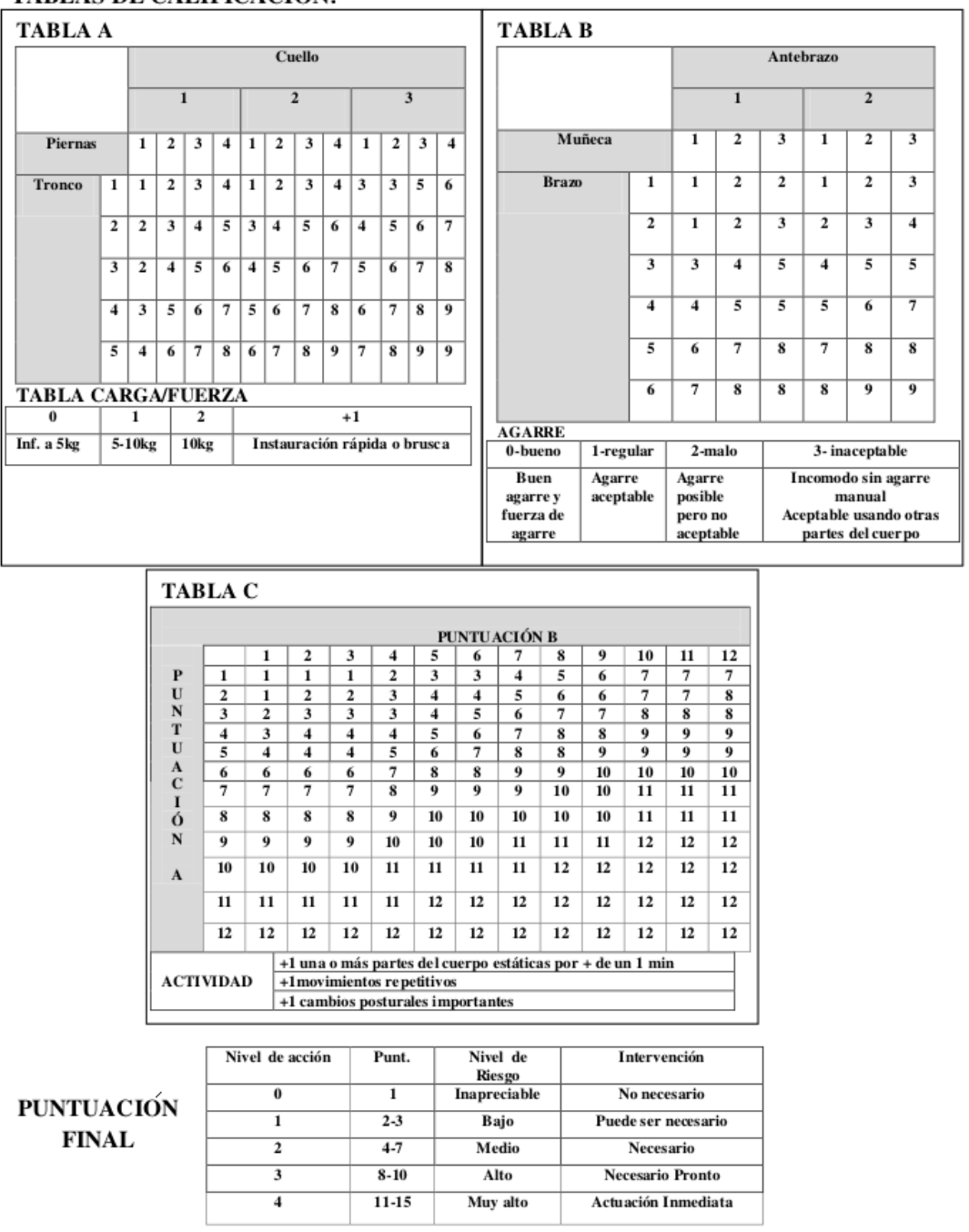

Fig. 2. Método R.E.B.A TABLAS DE CALIFICACIÓN

tuvo un nivel de riesgo medio de un total de 81 mujeres que participaron en el estudio. (Gráfico No.2).

En el sexo masculino en cambio se observó que ningún participante obtuvo un nivel de riesgo inapreciable en relación con el sexo femenino, es así que se encontró que el $45 \%$ (20) de varones tuvo un nivel de riesgo bajo y el $55 \%$ (24) de varones tuvo un nivel de riesgo medio de un total de 44 varones que participaron en el estudio. (Gráfico No.2).

\section{DISCUSIÓN}

En varias partes del mundo se han estudiado los niveles de riesgo de una postura dentro de la práctica odontológica, en nuestro estudio participaron 125 estudiantes donde se encontraron valores significativos, es así que se determinó que el $61.6 \%$ (77) presentó un nivel de riesgo medio por lo tanto la intervención es necesaria, el $37.6 \%$ (47) presentó un nivel de riesgo bajo, la intervención puede ser necesaria y el $0.8 \%(1)$ tuvo un nivel de riesgo inapreciable por lo tanto la intervención no es necesaria, a diferencia de nuestro estudio que presentó riesgos bajos y medios, Álvarez ${ }^{13}$ en el año de 2011 realizó una evaluación postural durante la atención clínica de pacientes, utilizando el Método REBA, se seleccionaron 40 estudiantes de la Universidad Nacional de Córdova-Argentina; obteniendo como resultado un $53 \%$ con un nivel de riesgo muy alto, un $42 \%$ con riesgo alto, riesgo medio un $5 \%$, Matute ${ }^{14}$ en el año 2009 investigó la frecuencia de las posturas inadecuadas en los estudiantes en 32 estudiantes de Odontología de la Universidad Autónoma de Nicaragua, utilizó el Método REBA, se determinó que el $90.62 \%$ tenía un nivel de riesgo muy alto,y el $9.37 \%$ con un nivel de riesgo alto, en cambio en nuestro estudio los valores de riesgo alto y muy alto no estuvieron presentes en 


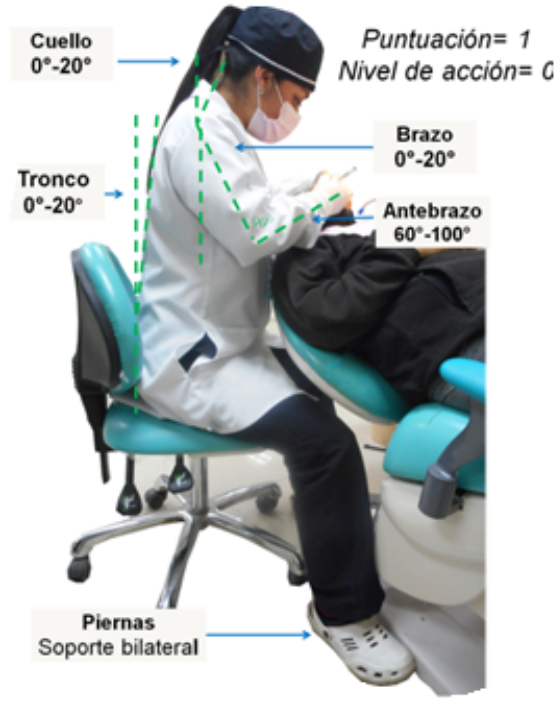

Fig. 3. Nivel de Riesgo = Inapreciable $/$ Intervención = No necesaria
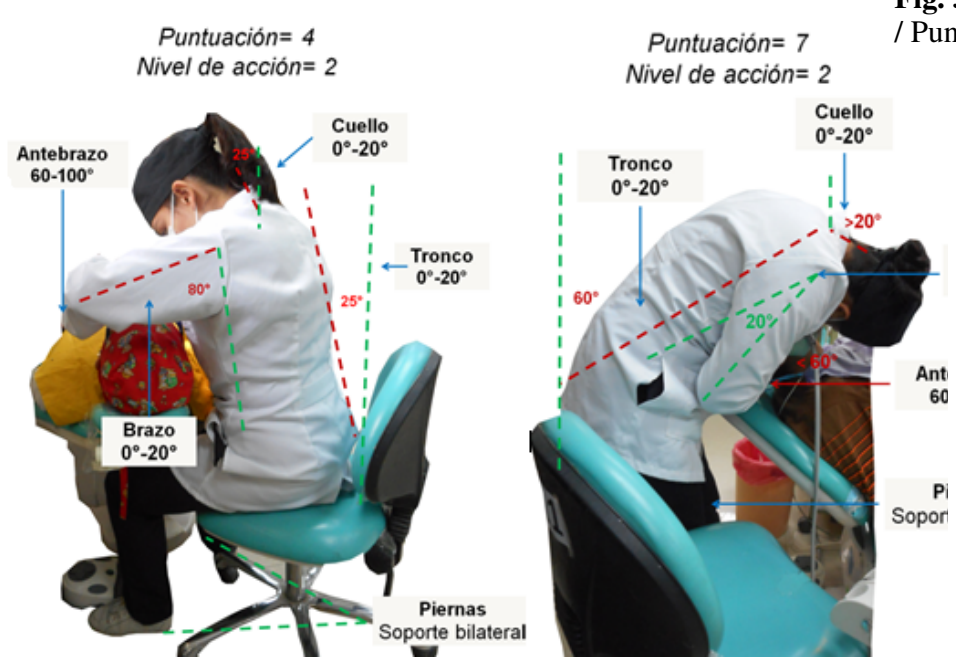

Fig. 4. Nivel de Riesgo = Medio/ Intervención $=$ Puede ser necesaria Puntuación $=4$ a 7

ningún estudiante, también Bolaños ${ }^{15}$ evaluó la postura de trabajo de 40 estudiantes de Quinto Curso de Odontología de la Universidad de Granada-España durante la realización de las prácticas clínicas sobre pacientes infantiles, el $89.7 \%$ presentó un riesgo postural medio donde fue necesario adoptar medidas correctoras, el $10.3 \%$ de los operadores tiene un nivel alto de riesgo requiriendo una pronta intervención , en nuestro estudio tambien se pudo encontrar un nivel de riesgo medio significativo con el $61.6 \%$, siendo el estudio de Bolaños el que mayor prevalencia de riesgo medio posee.

Los diversos estudios demuestran valores realmente preocupantes en cuanto a un nivel de riesgo en una postura de trabajo,a diferencia de nuestro estudio que presenta niveles

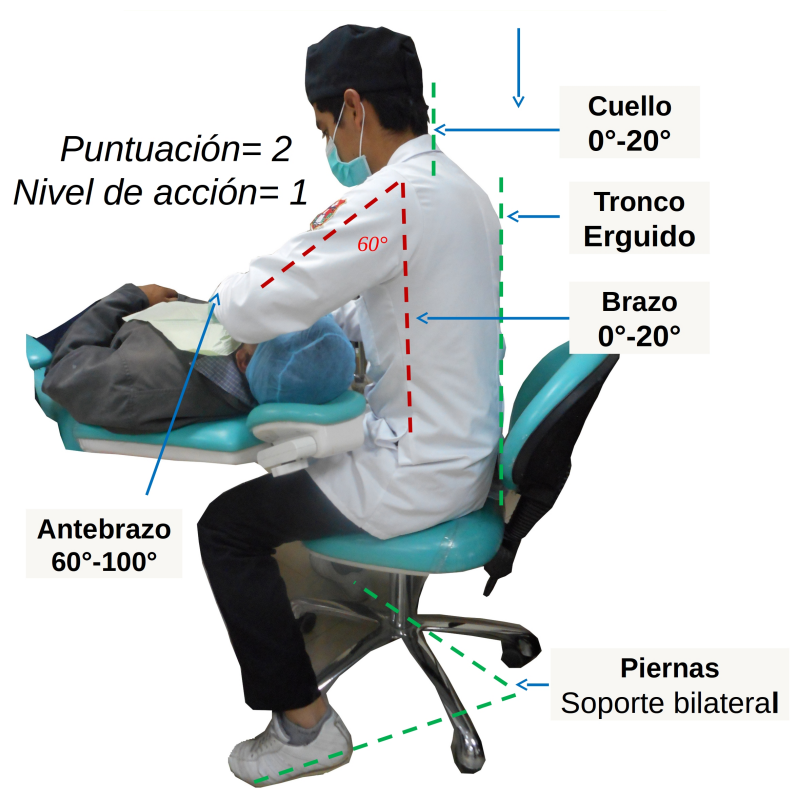

Fig. 5. Nivel de Riesgo $=$ Bajo $/$ Intervención $=$ Puede ser necesaria / Puntuación= 2 a 3

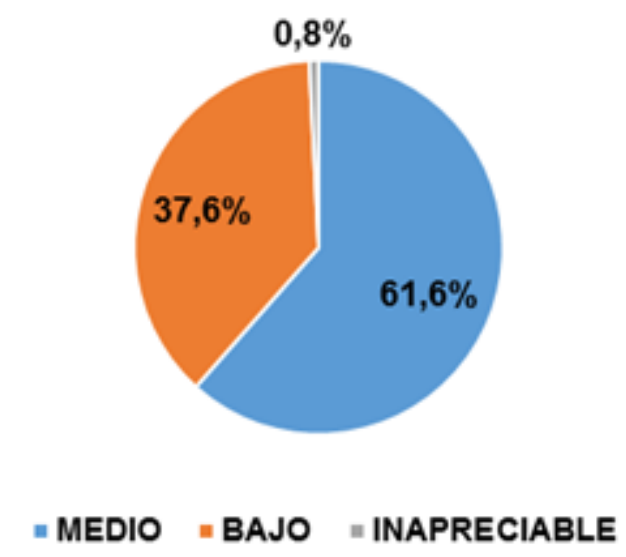

Grf. 1. Nivel de Riesgo de una postura bajos y medios de riesgo, la cantidad de personas en las cuales se aplicó el Método REBA es una de las diferencias más notorias al contar con la mayor cantidad de participantes en el estudio.

\section{CONCLUSIÓN}

Una inadecuada postura de trabajo fue evidenciada en los estudiantes con un nivel de riesgo medio (61.6\%) y un nivel de riesgo bajo $(37.6 \%)$, se recomienda evaluar el puesto de trabajo. La aplicación del método REBA en el desempeño de las actividades odontológicas nos permitió conocer las diversas posturas de trabajo de 


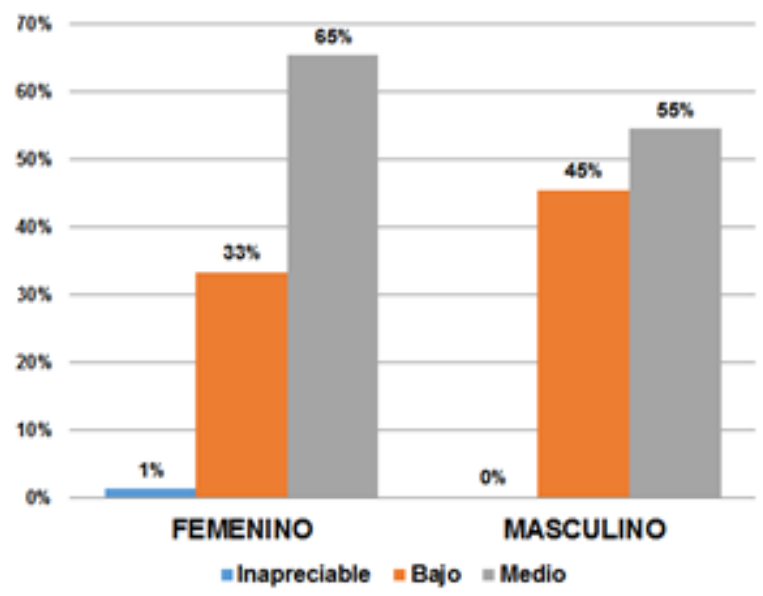

Grf. 2. Nivel de Riesgo de una postura
11 Angarita A et al. Revisión Sistemática sobre enfermedades laborales en odontología. Acta Bioclínica. p. 2-33, oct. 2014. ISSN 2244-8136. Documento disponible

12 Villavicencio-Caparó E. El tamaño muestral en tesis de post grado ¿Cuántas personas debo encuestar? Research Gate. [Online]. Arequipa; 2010 [Visitado 01 -enero-2016 Documento disponible

13 Álvarez, N, Gigena P. estrategia de intervención para disminuir el riesgo postural en estudiantes de odontología durante la atención clínica. Universidad nacional de Córdoba. Argentina. Huella: revista de vinculación con la comunidad de la Facultad de odontología 2014, vol. 2, no 4.

14 Matute C. Posturas de trabajo inadecuadas adoptadas por los estudiantes de 4to y 5to año de facultad de odontología UNAN - León, II semestre del año 2009. Cirujano Dentista. UNAN: Universidad Nacional Autónoma de Nicaragua; 2010.

15 Bolaños E. Evaluación de riesgos posturales en la clínica dental infantil. Odontol Pediátr.2004; Vol.12.N.2:72-79.

todos los estudiantes y evaluar el nivel de riesgo ergonómico.

\section{Referencias}

1 Singleton W. Objetivos Principios y Métodos. Wolfgang Laurig, Joachim Vedder. Enciclopedia de salud y seguridad en el trabajo.2010.Tomo 1.Cap29.p. 2-5.

2 Cortesi V. Manual práctico para el auxiliar de odontología. Barcelona: Elsevier España.S.L.2008.

3 Wonderley F, Mussolina de Queiroz A, Díaz K. Alteraciones Ocupacionales y su repercusión en el sistema estomatológico, Acta Odontológica Venezolana. 2008. ISSN: 0001 6365; Vol 46 no 4: 1 - 7 .

4 Cárdenas A, Sánchez F. Técnicas de ayuda Odontológica y Estomatológica. Ediciones Paraninfo, S.A. Segunda edición. 2013.

5 Moore K. Moore Anatomía con Orientación Clínica. Séptima Edición. Barcelona-España. Wolters Klumer Health. S.A. 2013. p. 439-471.

6 Bugarin R, Galego P, García A. Los trastornos musculo esqueléticos en los odontoestomatólogos. RCOE. 2005: Vol 10, no 5-6: 561-566.

7 Maldonado A, Ríos R, Quezada M, Análisis para determinar los factores que Inciden en los DTA'S que sufren los odontólogos. UANL. 2005: 22 - 32.

8 López T. Definición y Clasificación del Dolor. Madrid, 1996, Vol 4: 49-55.

9 Briones A. Posturas odontológicas ergonómicas y dolor muscular, durante las prácticas clínicas del Estudiante del 5to año de la facultad de Odontología periodo 2013. Título de Odontólogo. Guayaquil; Universidad de Guayaquil; 2014.

10 Hignett Sue, Mcatamney L. Rapid entire body assessment (REBA).Applied ergonomics.2000; vol.31- No.2: 201-205.
Recibido: 26 de Enero de 2016

Aceptado: 27 de Febrero de 2016 